\title{
Sequential and resonant field emission from nano-structured cathodes
}

\author{
L. D. Filip, M. Palumbo, J. D. Carey, and S. R. P. Silva \\ Advanced Technology Institute, University of Surrey, Guildford, \\ Surrey GU2 7XH, United Kingdom
}

Traditional field emission (FE) experiments have used a wide range of materials, which were initially exposed to pre-treatment procedures in order to burn off any residual contaminants present on the sample's surface. However, interesting results are obtained if the field emission experiment is performed on composite materials instead on pristine surfaces. For example, integrating alternating layers of dielectric and conductive materials or nanoparticles, the monotonic aspect of the current-voltage (I-V) curves is broken, giving rise to new field emission processes. The electron transport through nanometer-size objects is essentially influenced by the quantum confinement of the electronic wave function therein. The electron transport through heterostructures has been explained using a variety of quantum processes depending on the structure of the device. For instance, if a bulk conductor is covered by a dielectric layer and then a thin metallic layer is deposited (thin with respect to the mean free path of the electrons inside that metal), the electrons can in theory traverse this structure in a resonant manner thus creating sharp peaks in the FE diagram. However, if the dielectric layer is too thick (compared to the mean free path of the electrons) scattering events will destroy the coherence of the electron wave and the tunneling process will be described by a different mechanism. Consequently, it was shown that during FE from Au nano-particles step-like features are likely to appear due to the discrete nature of the energy levels inside the nano-particle. Such behavior was described using a two-step tunneling mechanism [1]. Nevertheless, it is clear that any given sample used in FE experiments which study a specific transport phenomena, is not perfect. Imperfections in the manufacturing process can create variations in the uniformity of the dielectric thickness, or if various impurities are present there can be regions with localized electronic states at the surface of this heterostructures. The results can thus in principle contain the signatures of a multitude of transport mechanisms for the electrons during FE and if only one is shown to be predominant in the data, the rest are disregarded as noise or due to the sample instability. This is a very plausible conclusion in an experiment, yet the current theories for electron emission do not consider the possibility that a mixture of transport mechanisms can happen at the same time during FE.

The present paper aims a unified point of view in this problem. We will attempt to integrate two electron transport mechanisms, by computing the FE current as a superposition of both resonant and sequential branches. More precisely, the supply current of electrons from the substrate is split into two parts: one that is going to pass coherently through the nano-object and the other that may reside for a while in the bound states therein. The fraction of the supply current that is to tunnel coherently is kept as a variable parameter to be fitted by comparison with real I-V diagrams. In order to keep the physics of the studied phenomenon as clear as possible, the quantum object through which the tunneling occurs is modeled as a one-dimensional (1D) potential well separated from the substrate by a potential barrier (See Figure 1). 


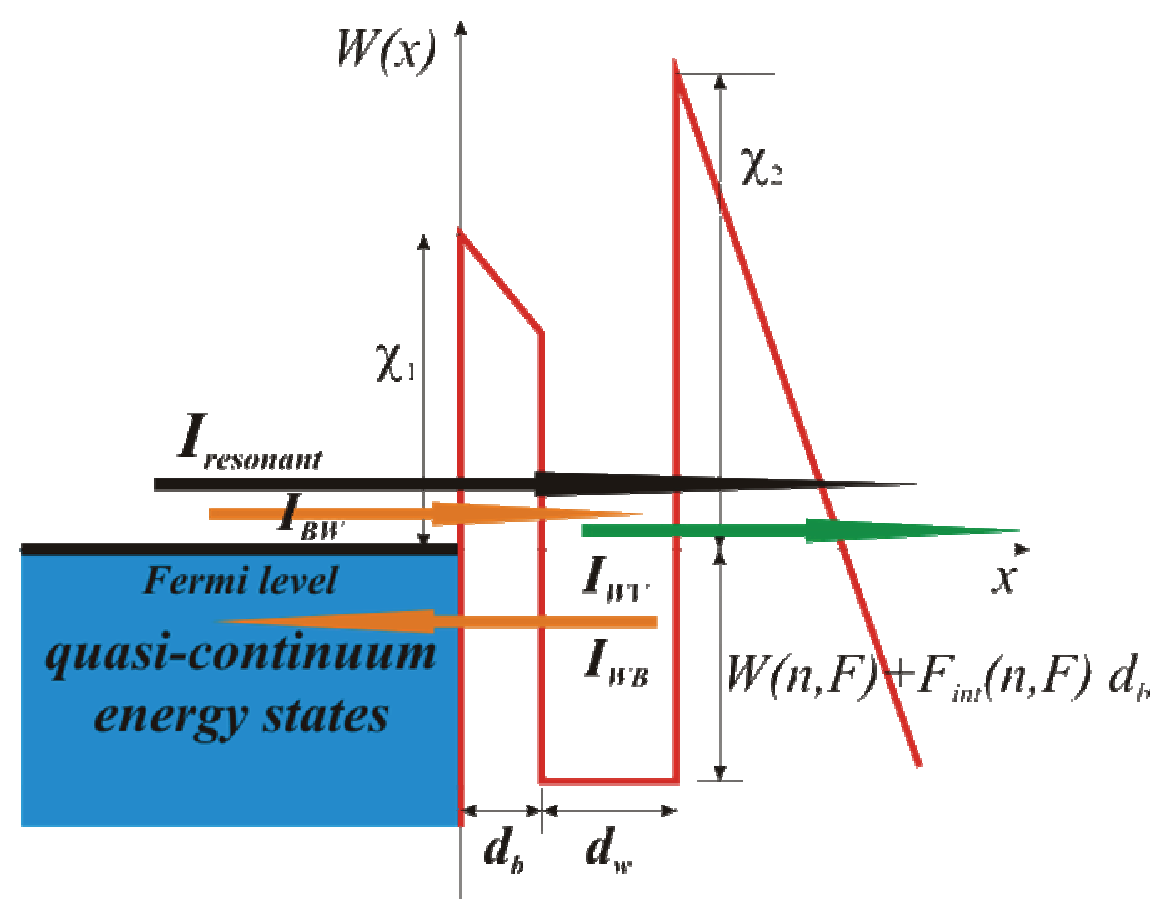

Figure 1: Sketch of the potential energy diagram for a heterostructure with an applied electric field.

The other side of the well is interfaced with the vacuum, where a uniform electric field is applied that produces a triangular output barrier. The field is considered to penetrate the wide-band-gap material that forms the barrier to the substrate. The model takes into account the changes in the electronic energies of the bound states in the well due to its transient charging during the process of sequential tunneling. There are two distinct mechanisms by which electrons pass through this structure toward vacuum: the resonant (or coherent) tunneling [2] and the sequential (or two-step) tunneling [1]. Resonant tunneling is generally associated to rather sharp singular peaks in the I-V characteristics [3]. On the other hand, the sequential tunneling mechanism is believed to produce ladder-shaped characteristics [1]. However, in many real cases both singular peaks and ladders appear in the same I-V characteristics. Hence, one may think that both tunneling mechanisms occur simultaneously.

The proposed model is useful in the interpretation of real I-V diagrams of FE through composite vacuum interfaces and in evaluating the amount of coherence in the related tunneling processes.

\section{References}

1. L.D. Filip, R.C. Smith, J.D. Carey, and S.R.P. Silva, Journal of Physics-Condensed Matter 21(19), 195302 (2009).

2. D. Dragoman and L.D. Filip, Journal of Applied Physics 99(9), 094307 (2006).

3. V. Litovchenko, A. Evtukh, Y. Kryuchenko, N. Goncharuk, O. Yilmazoglu, K. Mutamba, H.L. Hartnagel, and D. Pavlidis, Journal of Applied Physics 96(1), 867 (2004). 